\title{
Experiments on W-band extended interaction oscillator with pseudospark sourced post-accelerated electron beam
}

\author{
J. Zhao, ${ }^{1,2,3, a)}$ H. Yin, ${ }^{3}$ L. Zhang, ${ }^{3}$ W. He ${ }^{3}$ Q. Zhang, ${ }^{1,2}$ A. D. R. Phelps, ${ }^{3}$ and A. W. Cross ${ }^{3}$ \\ ${ }^{1}$ High Voltage Division, School of Electrical Engineering, Xi' an Jiaotong University, Xi' an 710049, China \\ ${ }^{2}$ State Key Laboratory of Electrical Insulation and Power Equipment, West Xianning Road, \\ $X i$ ' an 710049, China \\ ${ }^{3}$ Department of Physics, SUPA, University of Strathclyde, Glasgow, G4 ONG Scotland, United Kingdom
}

(Received 29 March 2017; accepted 26 May 2017; published online 8 June 2017)

The experimental study of a pseudospark discharge sourced electron beam with post acceleration (PA) is presented. The PA circuit was used to drive a W-band extended interaction oscillator. The experiments showed a significant increase in the output power, with $200 \mathrm{~W}$ at $94 \mathrm{GHz}$ measuring corresponding to a 5 times increase in the output power as compared to when post acceleration is not used. The microwave device developed has the advantage of not requiring an external magnetic field making it a low cost, compact, and portable sub-terahertz radiation source. Published by AIP Publishing. [http://dx.doi.org/10.1063/1.4985684]

Generation of microwave radiation at $100 \mathrm{GHz}$ to $3 \mathrm{THz}$ is a challenging technology, while high power, low cost, compact microwave sources in this frequency range are in great demand. In contrast to microwave vacuum electron devices based on conventional thermionic electron beam sources, the microwave radiation sources based on a pseudospark discharge can generate a high current density beam that does not require an external magnet field. ${ }^{1-3}$ In addition as the pseudospark discharge operates in the pulse mode, a compact pulse power supply can be used to drive it, ${ }^{4}$ resulting in a low cost portable sub-terahertz radiation source.

Microwave devices based on the pseudospark discharge sourced electron beam have been experimentally studied to cover various frequency bands, such as a Cherenkov maser operating at $\mathrm{Ka}$ band, ${ }^{5}$ an extended interaction oscillator (EIO) operating at W-band, ${ }^{6}$ and a backward wave oscillator (BWO) operating at G-band. ${ }^{7}$ It was found that the output microwave power is much smaller than the simulated values. A further study shows that the reduction in the radiation power is mainly caused by the beam velocity spread and the current lost during the beam propagating in the beam tunnel. One of the possible solutions is to use the post acceleration (PA) technology, in which the electron beam generated by the pseudospark discharge was then accelerated to a certain voltage by an extra electric field. The PA method has been demonstrated by Yin et al. ${ }^{8}$ The use of an additional power supply requires extra effort to synchronize the discharge pulse and the acceleration pulse. Recently, a simplified, compact PA circuit was developed that can be driven by a single power supply. ${ }^{9}$

In this paper, the experimental study on an EIO operating at $94 \mathrm{GHz}$ driven by the pseudospark discharge generated beam with PA is presented. By measuring the output radiation power, which was nearly 5 times higher than without PA, it shows a significant improvement on the beam propagation with the Extended Interaction Oscillator (EIO) with the PA technology.

\footnotetext{
a) Author to whom correspondence should be addressed: junping_zhao@ mail.xjtu.edu.cn
}

The pseudospark discharge sourced electron beam is with much higher current density compared with the thermionic cathode. When it propagates through the anode aperture, the beam ionizes the background gas to form a plasma channel and drives out part of the plasma electrons and only leaves the ions. The ion channel is able to stably transport the electron beam without the need of an external magnetic field if the ion density is sufficiently high. ${ }^{10}$

The beam current with higher energy is small, and a larger current with low energy is wasted as it does not satisfy the interaction condition. At the same time, from the plasma density ${ }^{11}$ and the gas pressure in the experiment, it is known that the background gas is partially ionized and it causes additional energy spread to the electron beam. It also causes the reduction in the beam current density during the propagation of the electron beam. For microwave devices, high energy spread will significantly affect the beam-wave interaction and reduce the output power.

A compact PA circuit has been developed. ${ }^{9}$ The schematic is shown in Fig. 1. It was used to accelerate the low energy, high current electron beam to the required voltage and to improve the beam-wave interaction. The experimental

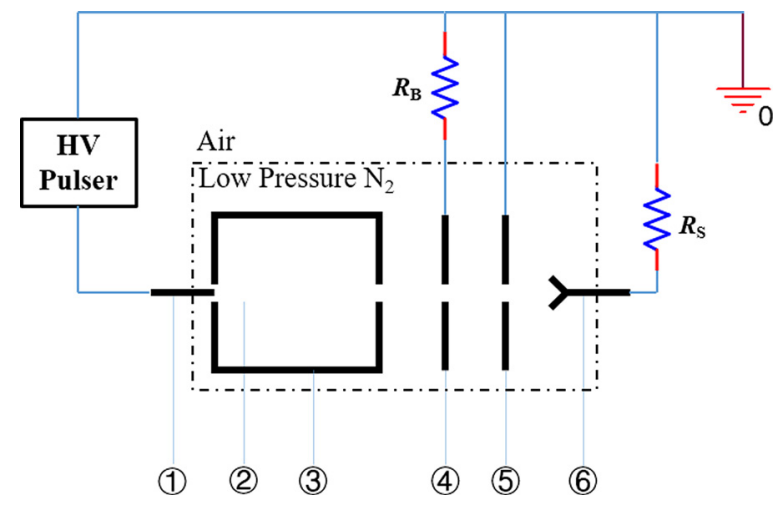

FIG. 1. Schematic diagram of the pseudospark discharge with post acceleration. (1) The trigger electrode, (2) pseudospark discharge cavity, (3) hollow cathode, (4) anode, (5) grounded electrode, and (6) Faraday cup for beam current measurements. 


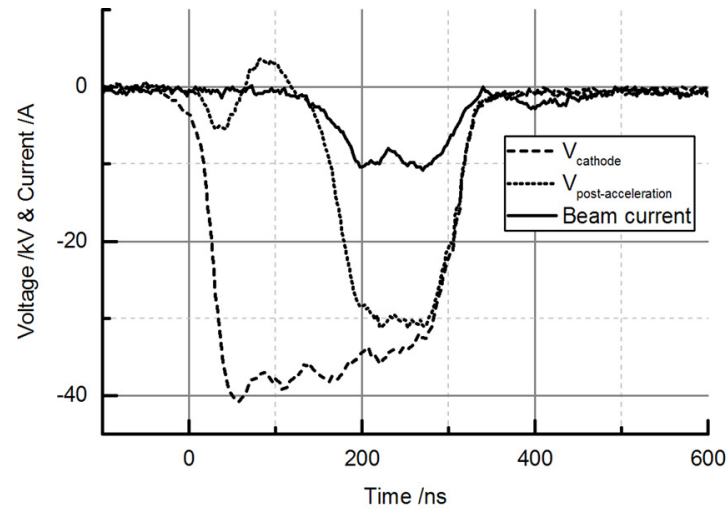

(a)

setup consists first the high voltage pulser, which is implemented by a pulse forming network (PFN) to generate the high voltage pulse to the trigger electrode (1). It injects seed electrons and initializes the pseudospark discharge. The anode (4) will be at the same high voltage as the cathode (3) after the breakdown between them. Then, the electron beam generated from the pseudospark discharge will be accelerated between the anode and the grounded electrode. A Faraday cup was used to measure the beam current, and it was later replaced by a phosphor screen to observe the beam image.

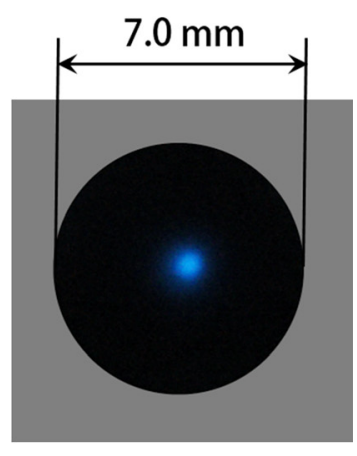

FIG. 2. Measurement results of the pseudospark discharge with PA. (a) Beam voltage, PA voltage, and beam current waveforms, and (b) electron beam image of the high energy component.

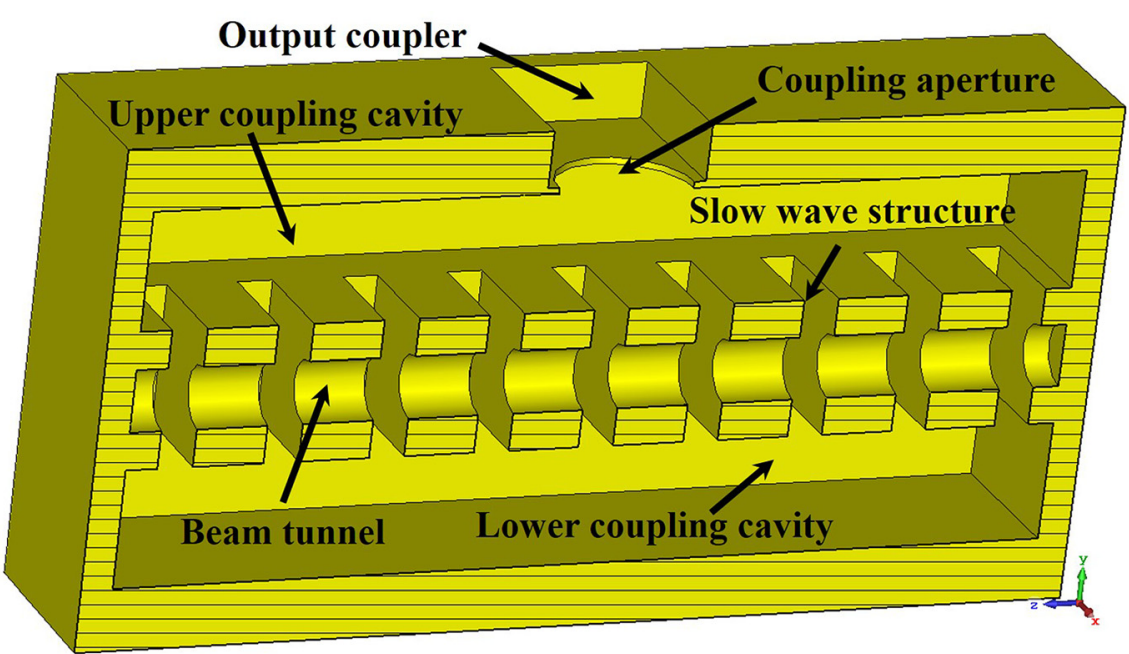

(a)

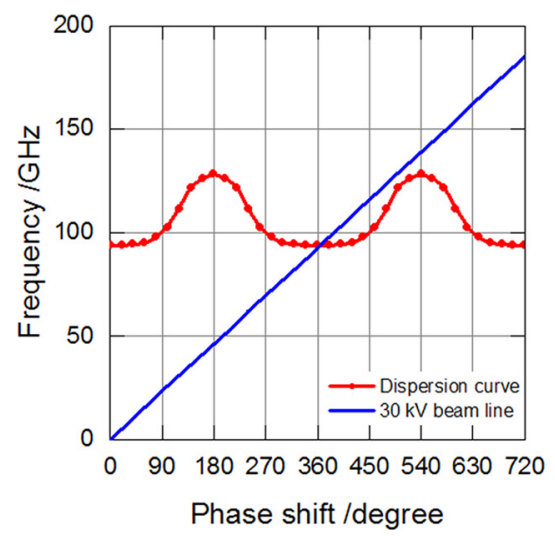

(b)

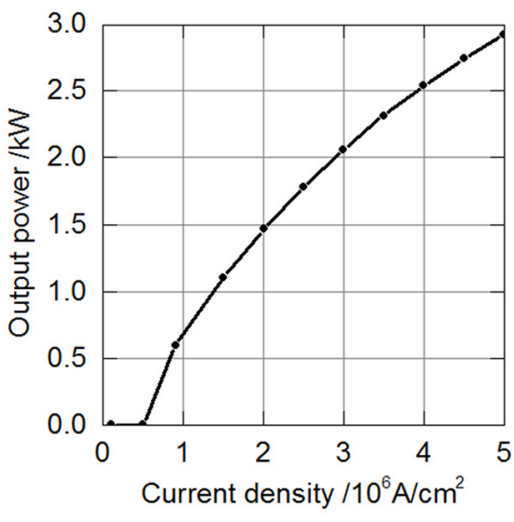

(c)
FIG. 3. (a) Structure of the EIO, (b) dispersion relations of the EIO with $30 \mathrm{kV}$ electron beam, and (c) the microwave power at different current densities. 
band EIO was designed to be driven by the pseudospark sourced post acceleration beam. Figure 3(a) shows the 3D geometry of the EIO structure. Two coupling cavities at the up and down sides form the extended resonance cavities. The center of the EIO structure is the beam tunnel with a diameter of $0.5 \mathrm{~mm}$. The EIO was designed to operate at the $2 \pi$ mode, and the synchronous condition for the beam wave interaction in this case is $v_{p}=L \cdot f \leq v_{e}$, where $L$ is the period of the EIO, $f$ is the operating frequency, and $v_{e}$ is the velocity of the electron beam, which can be calculated from the electron beam voltage. Therefore, $L \leq 1.05 \mathrm{~mm}$ when the operating frequency is $94 \mathrm{GHz}$. The cavity number was chosen to be nine as a result of balancing of the coupling coefficient and the overall length of the device. To reduce the complexity of the structure, all the nine cavities are identical. The dimensions of the cavity were further optimized to achieve maximum coupling impedance at the operating frequency of $94 \mathrm{GHz}$. The microwave radiation was coupled out from the middle cavity through a circular aperture, whose radius was optimized to achieve the maximum external quality factor $Q_{e}$. The details of the structure geometry and aspect ratio can be found in Ref. 6.

Figure 3(b) shows the dispersion curve of the EIO structure simulated by using 3D FDTD (Finite-Difference TimeDomain) code CST Microwave Studio. The desired beam voltage is $30 \mathrm{kV}$, and the interaction frequency is $94 \mathrm{GHz}$. The voltage range is about $\pm 3 \mathrm{kV}$ before it meets the undesired operating $2 \pi-1$ and $2 \pi+1$ modes. The performance of the EIO circuit was simulated by 3D particle-in-cell (PIC) code MAGIC. ${ }^{14}$ Figure 3(c) shows the output radiation power with different equivalent power densities. The output power reduces with the decrease in the current density. When the current density is higher than $5.5 \times 10^{6} \mathrm{~A} / \mathrm{cm}^{2}$, the output power only has slow increment. Doubling the current density only achieves about $10 \%$ more output power.

The whole EIO structure was divided into 3 pieces and machined by wire cutting technology and micromilling individually. A standard WR-10 waveguide was then connected to the EIO structure after assembly, and the reflection property was measured by a vector network analyzer (VNA). It shows a resonance frequency center at $93.8 \mathrm{GHz}$ which is fairly close to the design value.

The experimental configuration of the W-band EIO with the pseudospark discharge system is shown in Fig. 4. The EIO structure was inserted into a cylindrical tube to seal the vacuum. It was then connected to the grounded electrode of the PA circuit. The microwave radiation of the EIO was coupled out by a standard WR-10 waveguide connecting to a standard W-band $15 \mathrm{~dB}$ standard gain horn. A thin Mylar sheet was placed between the waveguide and horn to seal the vacuum. Another $15 \mathrm{~dB}$ gain horn was placed at a $20 \mathrm{~cm}$ distance to pick up a small proportion of the output radiation. The radiation power from the receiving horn was measured using a W-band crystal detector. Figure 5 shows the timecorrelated cathode voltage, PA voltage, and the microwave radiation pulse from the detector.

The detector was calibrated using a W-band $1.5 \mathrm{~W}$ solid-state source and a variable attenuator. The voltage responses from the detector were recorded at different input

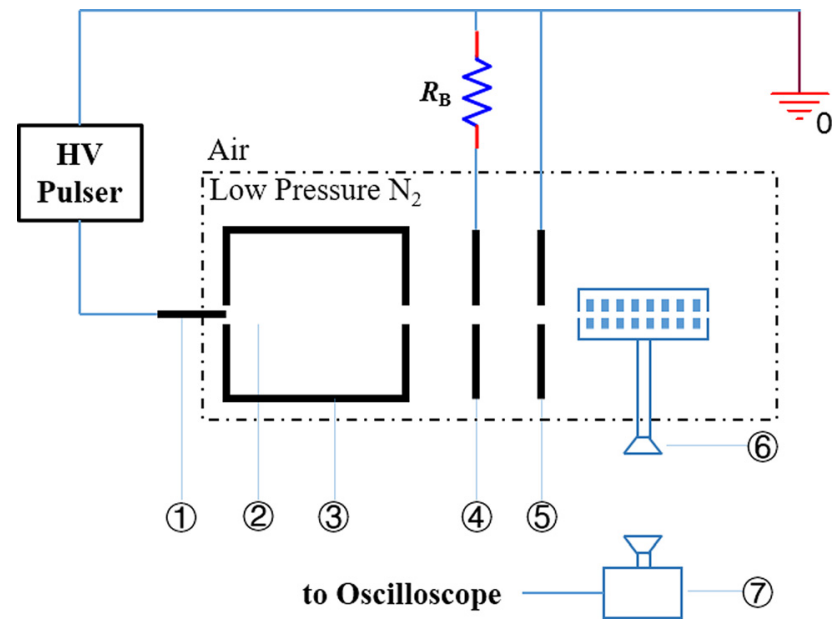

FIG. 4. Experimental setup of the pseudospark discharge system and W-band EIO experiment. (1)-(5) are the same as in Fig. 1. (6) EIO structure with WR-10 waveguide and pyramid horn, and (7) receiving horn and detector.

power levels and different distances between the transmitted and receiving horns. The calibrated data were then used to calculate the output radiation power from the EIO. The output power is measured to be $200 \mathrm{~W} \pm 20 \mathrm{~W}$.

As a comparison, experiments were also carried out without the PA by taking out the grounded electrode and connecting the anode to the ground. The output power with this setup was measured to be $40 \mathrm{~W} \pm 5 \mathrm{~W}$.

In order to determine the frequency of output radiation, cut-off filters were added between the W-band receiving horn and crystal detector. It was found that the radiation frequency is between 92 and $94 \mathrm{GHz}$, which is close to the simulations.

In this paper, a compact PA circuit for pseudospark discharge was developed and used to drive a W-band EIO. The experimental measurements show that the PA circuit is able to significantly increase the output microwave radiation power, from about $40 \mathrm{~W}$ without the PA to about $200 \mathrm{~W}$ with the PA circuit.

The microwave devices based on the pseudospark discharge generated beam have the advantage of not requiring an external magnetic field which makes them compact and

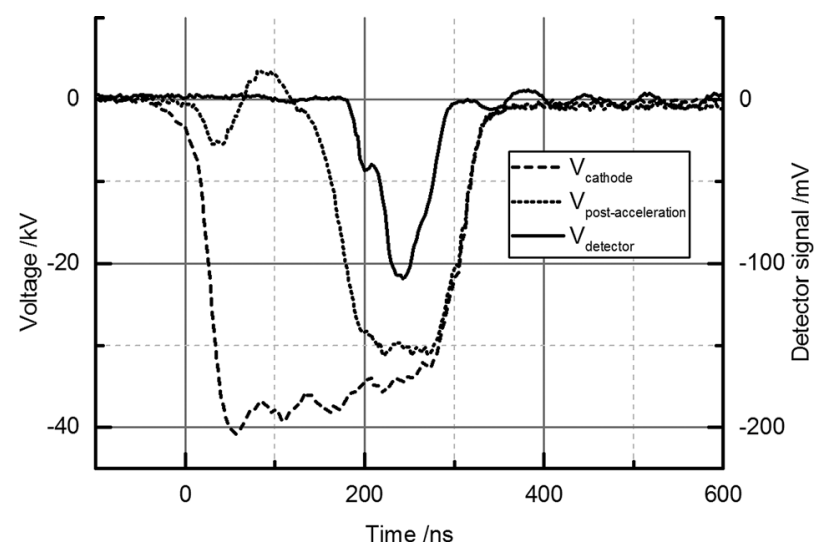

FIG. 5. Time-correlated electron beam voltage, current pulse, and the radiation pulse from the W-band EIO. 
low cost sources. With its high current density, the pseudospark discharge generated beam has the potential to be used for generating high microwave radiation at the mid-THz frequency range.

The authors would like to thank the Engineering and Physical Sciences Research Council (EPSRC) for supporting this work.

${ }^{1}$ J. Christiansen and C. Schultheiss, Z. Phys. A: At. Nucl. 290, 35 (1979).

${ }^{2}$ K. Frank and J. Christiansen, IEEE Trans. Plasma Sci. 17, 748 (1989).

${ }^{3}$ A. W. Cross, H. Yin, W. He, K. Ronald, A. D. R. Phelps, and L. C. Pitchford, J. Phys. D: Appl. Phys. 40, 1953 (2007).

${ }^{4}$ A. E. Dubinov, I. L. L. vov, S. A. Sadovoy, V. D. Selemir, D. V. Vyalykh, and V. S. Zhdanov, IEEE Trans. Plasma Sci. 40, 2079 (2012).

${ }^{5}$ H. Yin, A. W. Cross, W. He, A. D. R. Phelps, K. Ronald, D. Bowes, and C. W. Robertson, Phys. Plasmas 16, 063105 (2009).
${ }^{6}$ Y. Yin, W. He, L. Zhang, H. Yin, C. W. Robertson, and A. W. Cross, IEEE Trans. Electron Devices 63, 512 (2016).

${ }^{7}$ W. He, L. Zhang, D. Bowes, H. Yin, K. Ronald, A. D. R. Phelps, and A. W. Cross, Appl. Phys. Lett. 107, 133501 (2015).

${ }^{8}$ H. Yin, A. W. Cross, A. D. R. Phelps, D. Zhu, W. He, and K. Ronald, J. Appl. Phys. 91, 5419 (2002).

${ }^{9}$ J. Zhao, H. Yin, L. Zhang, G. Shu, W. He, Q. Zhang, A. D. R. Phelps, and A. W. Cross, Phys. Plasmas 24, 023105 (2017).

${ }^{10} \mathrm{~S}$. Liu, R. J. Barker, and Y. Yan, Theory of Wave Propagation Along Waveguide Filled with Moving Magnetized Plasma Waveguide (IEEE Press, 2000), p. 131.

${ }^{11}$ K. Takaharu, F. Tamiya, I. Minoru, I. Toshiaki, and W. Masayuki, Jpn. J. Appl. Phys. 44, 6747 (2005).

${ }^{12}$ J. Pasour, E. Wright, K. T. Nguyen, A. Balkcum, F. N. Wood, R. E. Myers, and B. Levush, IEEE Trans. Electron Devices 61, 1630 (2014).

${ }^{13}$ Y. Yin, W. He, L. Zhang, H. Yin, and A. W. Cross, Phys. Plasmas 22, 073102 (2015).

${ }^{14}$ B. Goplen, L. Ludeking, D. Smith, and G. Warren, Comput. Phys. Commun. 87, 54 (1995). 